\title{
Melatonin could Alleviate the Dysregulation of Metabolic Reprogramming in Periodontitis-Implications in Host Modulatory Therapy
}

Thodur M Balaji ${ }^{1}$, Saranya Varadarajan ${ }^{2}$, Debasish Bandyopadhyay ${ }^{3}$, Raghunathan Jagannathan ${ }^{4}$, CJ Venkatakrishnan $^{5}$, Ashok K Bhati ${ }^{6}$, A Thirumal Raj $^{7}$, Swaminathan Rajendiran ${ }^{8}$, Shankargouda Patil ${ }^{9}$

\begin{abstract}
Periodontitis is an infectious disease of the tooth-supporting apparatus characterized by connective tissue breakdown and alveolar bone resorption ultimately resulting in tooth loss. A chronic immune response and jeopardized oxidant-antioxidant balance are cardinal features in the pathobiology of periodontitis. The resident cells of the periodontium are known to undergo metabolic changes in the pathogenesis of periodontitis. The principal cellular fraction of the periodontal ligament space in states of health, disease, repair, and regeneration is the periodontal ligament stem cells (PDLSCs). Although these cells are believed to adapt well to bacterial infections, a recent in vitro study has shed light on the metabolic changes in these stem cells infected with Porphyromonas gingivalis lipopolysaccharide. The findings of the study demonstrated elevated levels of Krebs cycle enzymes, succinate, and hypoxia-inducible factor 1 alpha (HIF-alpha) in the stem cells following $P$. gingivalis infection. In this context, we hypothesize a potential role that could be played by melatonin, an indoleamine molecule that has been found to play a significant role in periodontal homeostasis. It has been proposed that exogeneous melatonin supplementation in periodontitis could help in targeting metabolic dysregulation as melatonin is endowed with potent anti-inflammatory and antioxidant properties. Melatonin could also help in decreasing succinate production in the PDLSC by increasing alpha-ketoglutarate generation and could inhibit stabilization of HIF-alpha. Melatonin-mediated conversion of proinflammatory M1 macrophage to anti-inflammatory M2 macrophage phenotype could help in the resolution of periodontal disease and foster healing mechanisms in the diseased periodontium.
\end{abstract}

Keywords: Hypothesis, Melatonin, Metabolic dysregulation, Periodontitis.

World Journal of Dentistry (2021): 10.5005/jp-journals-10015-1823

\section{INTRODUCTION}

The human periodontium is a composite structure that forms the tooth-supporting attachment apparatus. This complex tissue composed of the avascular cementum, fibrous periodontal ligament, and alveolar bone is cased in the gingiva (gums) which is a soft tissue forming an integral part of the oral mucosa. ${ }^{1}$ Accumulation of dental plaque, a soft microbial biofilm around the gingival margins causes inflammation of the gingival tissues denoted as gingivitis. ${ }^{2}$ Untreated gingivitis in some but not all cases extends to involve the periodontal attachment apparatus consequently leading to an irreversible condition called periodontitis. Periodontitis is clinically characterized by the formation of gum pockets that bleed upon probing. In some cases, this condition is associated with the formation of periodontal abscesses and tooth mobility which consequently leads to tooth loss. ${ }^{3}$ Periodontitis is a disease with significant systemic impact as it has been proposed as a risk factor for cardiovascular disease, ${ }^{4}$ diabetes mellitus type $2,{ }^{5}$ and adverse pregnancy outcomes. ${ }^{6}$

Regarding the pathogenesis of periodontitis, it has been understood that microbial challenge from the subgingival dental plaque biofilm ${ }^{7}$ elicits a chronic inflammatory and immune response in the host periodontal tissues ${ }^{8,9}$ coupled with an overzealous production of reactive oxygen species (ROS). ${ }^{10}$ The component cells of the periodontium like the epithelial cells, fibroblasts, macrophages, bone cells, and periodontal ligament cells are believed to undergo metabolic changes in the pathogenesis of periodontitis due to a dysregulated immune and redox response as abovementioned. This phenomenon of metabolic reprogramming
1Department of Dentistry, Bharathirajaa Hospital and Research Institute, Chennai, Tamil Nadu, India

${ }^{2,7}$ Department of Oral Pathology and Microbiology, Sri Venkateswara Dental College and Hospital, Chennai, Tamil Nadu, India

${ }^{3}$ Department of Physiology, Oxidative Stress and Free Radical Biology Laboratory, University of Calcutta, University College of Science and Technology, Kolkata, West Bengal, India

${ }^{4}$ Department of Periodontics, Tagore Dental College and Hospital, Chennai, Tamil Nadu, India

${ }^{5}$ Department of Prosthodontics, Tagore Dental College and Hospital, Chennai, Tamil Nadu, India

${ }^{6}$ Division of Periodontics, College of Dentistry, Jazan University, Jazan, Kingdom of Saudi Arabia

${ }^{8}$ Department of Periodontics, Sri Venkateswara Dental College and Hospital, Chennai, Tamil Nadu, India

${ }^{9}$ Department of Maxillofacial Surgery and Diagnostic Sciences, Division of Oral Pathology, College of Dentistry, Jazan University, Jazan, Kingdom of Saudi Arabia

Corresponding Author: Shankargouda Patil, Department of Maxillofacial Surgery and Diagnostic Sciences, Division of Oral Pathology, College of Dentistry, Jazan University, Jazan, Kingdom of Saudi Arabia, Phone: +966507633755, e-mail: dr.ravipatil@gmail.com

How to cite this article: Balaji TM, Varadarajan S, Bandyopadhyay $D$, et al. Melatonin could Alleviate the Dysregulation of Metabolic Reprogramming in Periodontitis-Implications in Host Modulatory Therapy. World J Dent 2021;12(2):166-170.

Source of support: Nil

Conflict of interest: None

() Jaypee Brothers Medical Publishers. 2021 Open Access This article is distributed under the terms of the Creative Commons Attribution 4.0 International License (https://creativecommons.org/licenses/by-nc/4.0/), which permits unrestricted use, distribution, and non-commercial reproduction in any medium, provided you give appropriate credit to the original author(s) and the source, provide a link to the Creative Commons license, and indicate if changes were made. The Creative Commons Public Domain Dedication waiver (http://creativecommons.org/publicdomain/zero/1.0/) applies to the data made available in this article, unless otherwise stated. 
at the tissue level has been found to occur in many systemic conditions. ${ }^{11}$ But a recently performed study on periodontal ligament stem cells (PDLSCs) in the periodontal ligament found significant changes. ${ }^{12}$ Periodontal ligament stem cells are a mesenchymal stem cell population that forms a principal cellular fraction of the periodontal ligament space in states of health, disease, repair, and regeneration. These cells form and stabilize extracellular matrix components and due to their stemness and plastic properties, are believed to adapt well to bacterial infections. ${ }^{13}$ Periodontal ligament stem cells are equipped with a wide array of pattern recognition receptors (PRRs), such as toll-like receptors (TLRs) and CD36, to sense the invading microbials, undergo fundamental changes in cell biology, and transmit danger signals to defense cells. ${ }^{14}$ The interaction of PDLSC with macrophages in the periodontal ligament space and their influence on macrophage polarization from M1 (proinflammatory phenotype) to $\mathrm{M} 2$ (anti-inflammatory phenotype) subtypes has been previously documented. ${ }^{15}$ In a recently performed in vitro study, it was found that infection of human PDLSC with Porphyromonas gingivalis lipopolysaccharide caused significant molecular and biochemical changes. ${ }^{12}$ Porphyromonas gingivalis infection led to a shift in metabolic reprogramming from mitochondrial oxidative phosphorylation to cytosolic glycolysis in the PDLSC. This aberrant change has been termed by researchers as the Warburg effect which is classically defined as a phenomenon where pyruvate is used by cells as a metabolic substrate to release quick energy by aerobic glycolysis mechanism. ${ }^{16}$ The Warburg effect has been documented to occur typically in cancer cells to acquire quick energy to undergo uncontrolled proliferation. ${ }^{17}$ In the periodontal ligament cells also, an aberrant metabolic dysregulation was reported by the abovementioned study following bacterial infection. Profiling of the enzymes revealed that $P$. gingivalis infection of the PDLSC caused increased expression of the Krebs cycle enzymes isocitrate dehydrogenase, succinic dehydrogenase, and citrate synthase. It was found that the infected PDLSC accumulated high levels of succinate. The source of succinate in the PDLSC was contemplated to be derived through glutamine-dependent anaplerosis and the $\gamma$-aminobutyric acid (GABA) shunt although not demonstrated. ${ }^{12}$ The findings of increased succinate dehydrogenase in the periodontal context are different from other in vitro models. In monocytes infected with LPS, it has been found that succinate elevation was coupled with dampened succinic dehydrogenase levels, ${ }^{18}$ whereas $P$. gingivalis infection of PDLSC led to upregulated succinic dehydrogenase. The consequence of succinate elevation in the PDLSC was its export into the cytosol from the mitochondria which caused stabilization of hypoxia-inducible factor 1 alpha (HIF-alpha). ${ }^{12}$ This situation in the PDLSC was found to create a pseudohypoxia-like situation and was found to turn on the transcription of proinflammatory cytokine genes. It was also observed that $P$. gingivalis infection of PDLSC caused a significant elevation of intracellular ROS. ${ }^{12}$ The abovementioned novel experiment has pointed out some intricate metabolic changes at the cellular and molecular levels in periodontal disease. We hypothesize that melatonin administered exogenously could mitigate the deleterious effects of metabolic reprogramming that occurs in the periodontal tissues through multiple mechanisms. It is well known that endogenous melatonin plays a significant role in the pathobiology of periodontal disease. Melatonin also termed $\mathrm{N}$-acetyl-5 methoxytryptamine is a product of tryptophan metabolism that is synthesized predominantly by the pinealocytes of the pineal gland..$^{19}$ It has been demonstrated that tissues other than the pineal gland could also synthesize and release melatonin. In the oral cavity, the salivary glands ${ }^{20}$ and the gingiva ${ }^{21}$ are endowed with the capacity of melatonin synthesis and also bear receptors for melatonin. ${ }^{21}$ Melatonin receptors have been found on the gingival epithelial cells, gingival fibroblasts, and immune and inflammatory cells in the gingival connective tissues. ${ }^{21}$ Melatonin has been found to perform numerous homeostatic functions in the human body. However, its depletion in periodontal disease necessitates exogenous supplementation which would exert profound effects as described below.

\section{The Hypothesis}

Melatonin administration could exert the following beneficial cellular and molecular effects in the periodontium. It is to be reiterated that it is not known if melatonin receptors are present on PDLSC. But the hypothesis still holds good based on the fact that melatonin exerts both receptor-independent and receptordependent effects. Hence, the below-mentioned effects of melatonin could be achieved in the PDLSC context even if melatonin receptors are not present on them.

- Decreasing succinate production by increasing alphaketoglutarate generation in the PDLSC followed by exosomal extrusion of the alpha-ketoglutarate into the periodontal microenvironment.

- Reducing the effects of succinate-mediated HIF-alpha stabilization in the PDLSC by dephosphorylation of p70S6K and its direct target RP-S6 by repressing the mammalian target of mTORC1.

- Potentially reducing the levels of ROS and NF kappa B in the PDLSC through antioxidant and anti-inflammatory mechanisms.

- Contributing to the conversion of the proinflammatory M1 macrophage phenotype to the anti-inflammatory M2 macrophage phenotype through the utilization of the alphaketoglutarate exosomes produced by PDLSC.

\section{Melatonin and Its Association with Succinate and Alpha-ketoglutarate Production}

Melatonin is a potent inhibitor of the Warburg effect which operates in cancer cells causing uncontrolled proliferation. ${ }^{22}$ About elevated succinate, in PDLSC, the phenomenon was pointed out to occur due to glutamine-dependent anaplerosis and GABA shunt akin to macrophages challenged with LPS. At this point, the role of melatonin can be clearly explained. Melatonin has been found to increase glutaminolysis, thereby converting glutamine to glutamate in the adipose tissue-derived macrophage model. ${ }^{23}$ The generated glutamate is further transported from the cytosol into the mitochondria to become alpha-ketoglutarate. The generated alpha-ketoglutarate under the influence of melatonin is packed into exosomes and extruded by the cells into the surrounding microenvironment. By this mechanism of alpha-ketoglutarate utilization, succinate generation could be countered by melatonin and its accumulation in the cytosol is also prevented. The exosomal extrusion of alpha-ketoglutarate has been demonstrated to occur in adipose tissue under the influence of melatonin where the adipocyte-derived alpha-ketoglutarate has been extruded into the microenvironment as exosomes. ${ }^{24}$ 


\section{Effects of Melatonin on Succinate- mediated Hif-alpha Stabilization}

As described earlier, the elevated succinate in the PDLSC consequently causes HIF-alpha stabilization and proinflammatory gene transcription. This mechanism could also be repressed by melatonin through dephosphorylation of p70S6K and its direct target RP-S6 by repressing the mammalian target of mTORC1. ${ }^{23}$ By this described pathway, melatonin destabilizes HIF-alpha in the macrophage model. In the same method, melatonin could destabilize HIF-alphas in the PDLSC, thereby reversing the pseudohypoxic situation and downregulating HIF-alpha mediated proinflammatory gene transcription.

\section{Influence of Melatonin on Intracellular ROS Production and NF Kappa B Activity}

The $P$. gingivalis challenge was also found to increase intracellular ROS levels in the PDLSC. This reaction can be efficiently countered by melatonin as it is a powerful antioxidant under in vitro and in vivo conditions. ${ }^{25}$ Several studies have been performed to demonstrate the antioxidant potential of melatonin. It has been shown that melatonin is superior to conventional antioxidants as one molecule of melatonin could scavenge up to ten ROS molecules. ${ }^{26}$ Melatonin and its metabolites AMK and AFMK are endowed with the capacity of scavenging the most lethal hydroxyl radical implicated in many pathological conditions. ${ }^{27}$ About NF kappa B, melatonin has been found as a potent inhibitor of this transcription factor. ${ }^{28}$ An experiment on melatonin treatment found a reduction in NF kappa B production in $P$. gingivalis fimbriae-treated monocytes showing the anti-inflammatory effects of melatonin. ${ }^{29}$ In addition to its antioxidant and anti-inflammatory effects, melatonin is also a cytoprotective agent. ${ }^{30}$ Hence, it is expected that melatonin administration could exert antioxidant, anti-inflammatory, and cytoprotective effects on the PDLSC.

\section{Effects of Melatonin on Macrophage Polarization}

The exosomal transport of alpha-ketoglutarate from the PDLSC as previously described could further be taken up by macrophages in the periodontal microenvironment resulting in a polarization of macrophage phenotype from destructive $\mathrm{M} 1$ to a reparative M2 phenotype. ${ }^{23}$ This kind of mechanism has been demonstrated under the influence of melatonin in adipose tissue where melatonin inhibits adipose inflammation through alpha-ketoglutarate. ${ }^{24}$ There is enough body of evidence available to demonstrate the interaction and cross-talk between macrophages and PDLSC in states of periodontal health and disease. ${ }^{31}$ Hence, this cross-talk could be efficiently modulated by melatonin administration which could favor a polarization of the macrophages toward the $M 2$ phenotype with anti-inflammatory properties. Figure $1 \mathrm{~A}$ summarizes the mechanisms of periodontal destruction, while Figure $1 \mathrm{~B}$ summarizes how these mechanisms could be countered with an exogenous supplementation of melatonin.

\section{Testing the Hypothesis}

It would be worthwhile testing the positive influence of melatonin on PDLSC infected with $P$. gingivalis in an in vitro model. Since it is not known if PDLSC bears melatonin receptors, it would be worthwhile to assess if melatonin receptors are present in these cells by immunohistochemical techniques. Succinate, alpha-ketoglutarate, and HIF-alpha levels should be tested in PDLSC-infected cultures at baseline and following melatonin treatment to assess if melatonin exerts positive effects on metabolic reprogramming. Animal studies could be conducted to assess if melatonin treatment in a model of ligature-induced periodontitis could improve cross-talk between PDLSC and macrophages favoring a polarization from M1 to the M2 phenotype. If in vitro and animal studies yield positive results, human clinical studies in this direction can be performed.

\section{Clinical Implications}

Taking all the available evidence into account, melatonin could be used as an efficient host modulatory agent in periodontal therapy. It would be worthwhile to assess the efficacy of melatonin in the systemic formulation and topical formulations such as mouthwashes, gels, gummies, and toothpastes in controlling metabolic reprogramming in the periodontal tissues. It is previously known that the oral tissues bear melatonin receptors. Hence, melatonin could exert both receptor-independent and receptormediated effects in the management of the periodontal disease.

\section{References}

1. Nanci A, Bosshardt DD. Structure of periodontal tissues in health and disease*. Periodontol 2000 2006;40(1):11-28. DOI: 10.1111/j.16000757.2005.00141.x.

2. Löe $H$, Theilade $E$, Jensen SB. Experimental gingivitis in man. J Periodontol 1965;36(3):177-187. DOI: 10.1902/jop.1965.36.3.177.

3. Armitage GC, Cullinan MP. Comparison of the clinical features of chronic and aggressive periodontitis. Periodontol 2000 2010;53(1):1227. DOI: 10.1111/j.1600-0757.2010.00353.x.

4. Scannapieco FA. Position paper of the American Academy of Periodontology: periodontal disease as a potential risk factor for systemic diseases. J Periodontol 1998;69:841-850. Im Internet: http:// www.ncbi.nlm.nih.gov/pubmed/9706864.

5. Grossi SG, Genco RJ. Periodontal disease and diabetes mellitus: a two-way relationship. Ann Periodontol 1998;3(1):51-61. DOI: 10.1902/ annals.1998.3.1.51.

6. Offenbacher $S$, Katz V, Fertik G, et al. Periodontal infection as a possible risk factor for preterm low birth weight. J Periodontol 1996;67(10s):1103-1113. DOI: 10.1902/jop.1996.67.10s.1103.

7. Bodet C, Chandad F, Grenier D. Potentiel pathogénique de porphyromonas gingivalis, Treponema denticola et Tannerella forsythia, le complexe bactérien rouge associé à la parodontite. Pathol Biol 2007;55(3-4):154-162. DOI: 10.1016/j.patbio.2006.07.045.

8. Kinane DF, Lappin DF. Immune processes in periodontal disease: a review. Ann Periodontol 2002;7(1):62-71. DOI: 10.1902/ annals.2002.7.1.62.

9. Page RC. The pathobiology of periodontal diseases may affect systemic diseases: inversion of a paradigm. Ann Periodontol 1998;3(1):108-120. DOI: 10.1902/annals.1998.3.1.108.

10. Wang $Y$, Andrukhov O, Rausch-Fan X. Oxidative stress and antioxidant system in periodontitis. Front Physiol 2017. 8. DOI: 10.3389/ fphys.2017.00910.

11. Zhang H, Wang D, Li M, et al. Metabolic and proliferative state of vascular adventitial fibroblasts in pulmonary hypertension is regulated through a MicroRNA-124/PTBP1 (polypyrimidine tract binding protein 1)/pyruvate kinase muscle axis. Circulation 2017;136(25):2468-2485. DOI: 10.1161/CIRCULATIONAHA.117.028069.

12. Su W, Shi J, Zhao Y, et al. Porphyromonas gingivalis triggers inflammatory responses in periodontal ligament cells by succinatesuccinate dehydrogenase-HIF-1a axis. Biochem Biophys Res Commun 2020;522(1):184-190. DOI: 10.1016/j.bbrc.2019.11.074. 


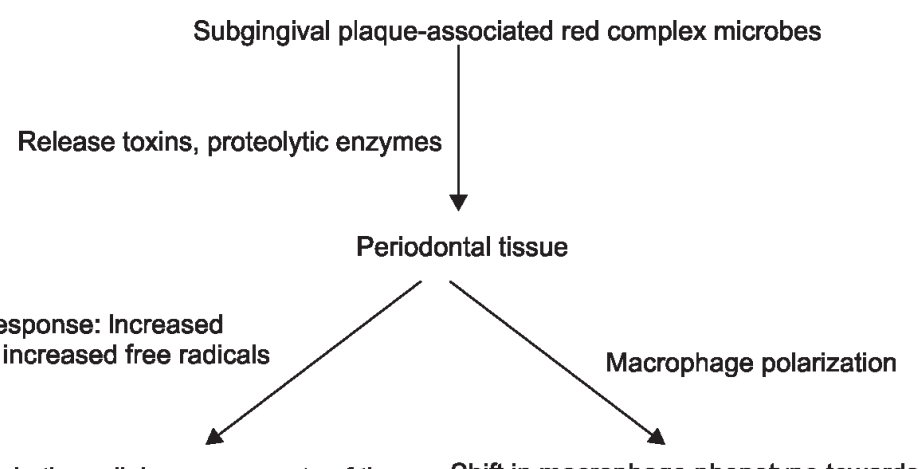

proinflammatory cytokines, increased free radicals

Metabolic dysregulation in the cellular components of the periodontium including the periodontal ligament stem cells

Shift in macrophage phenotype towards the M1 subtype (proinflammatory)

Warburg effect: Switch from mitochondrial oxidative phosphorylation to cytosolic glycolysis Increase in glutathione-dependent anaplerosis and GABA shunt

is

Increased accumulation of succinate within the cells mitochondria, which is in turn exported into the cytosol

Stabilizes hypoxia-inducible factor-1 alpha

A

Periodontal tissue destruction

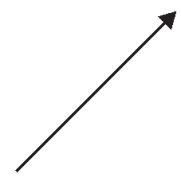


13. Zhu W, Liang M. Periodontal ligament stem cells: current status, concerns, and future prospects. Stem Cells Int 2015;2015:1-11. DOI: $10.1155 / 2015 / 972313$

14. Morandini ACF, Chaves Souza PP, Ramos-Junior ES, et al. Toll-like receptor 2 knockdown modulates interleukin (IL)- 6 and IL-8 but not stromal derived factor-1 (SDF-1/CXCL12) in human periodontal ligament and gingival fibroblasts. J Periodontol 2013;84(4):535-544. DOI: 10.1902/jop.2012.120177.

15. Liu J, Chen B, Bao J, et al. Macrophage polarization in periodontal ligament stem cells enhanced periodontal regeneration. Stem Cell Res Ther 2019;10(1):320. DOI: 10.1186/s13287-019-1409-4.

16. Warburg $\mathrm{O}$. The metabolism of carcinoma cells. J Cancer Res 1925;9(1):148-163. DOI: 10.1158/jcr.1925.148.

17. Liberti MV, Locasale JW. The Warburg effect: how does it benefit cancer cells? Trends Biochem Sci 2016;41(3):211-218. DOI: 10.1016/j. tibs.2015.12.001.

18. Lachmandas E, Boutens L, Ratter JM, et al. Microbial stimulation of different toll-like receptor signalling pathways induces diverse metabolic programmes in human monocytes. Nat Microbiol 2017;2(3):16246. DOI: 10.1038/nmicrobiol.2016.246.

19. Reiter RJ. Pineal melatonin: cell biology of its synthesis and of its physiological interactions. Endocr Rev 1991;12(2):151-180. DOI: 10.1210/edrv-12-2-151.

20. Shimozuma M, Tokuyama R, Tatehara S, et al. Expression and cellular localizaion of melatonin-synthesizing enzymes in rat and human salivary glands. Histochem Cell Biol 2011;135(4):389-396. DOI: 10.1007/ s00418-011-0800-8.

21. Madapusi BT, Rao SR. Preliminary evaluation of human gingiva as an extrapineal site of melatonin biosynthesis in states of periodontal health and disease. J Clin Diagnostic Res 2018;12:ZF01-ZF07. DOI: 10.7860/JCDR/2018/32451.11078.

22. Mao L, Dauchy RT, Blask DE, et al. Melatonin suppression of aerobic glycolysis (Warburg effect), survival signalling and metastasis in human leiomyosarcoma. J Pineal Res 2016;60(2):167-177. DOI: 10.1111/jpi.12298.
23. Xia $Y$, Chen $S$, Zeng $S$, et al. Melatonin in macrophage biology: current understanding and future perspectives. J Pineal Res 2019;66(2):e12547. DOI: 10.1111/jpi.12547.

24. Liu Z, Gan L, Zhang T, et al. Melatonin alleviates adipose inflammation through elevating a-ketoglutarate and diverting adipose-derived exosomes to macrophages in mice. J Pineal Res 2018;64(1):e12455. DOI: 10.1111/jpi.12455.

25. Ghosh AK, Naaz S, Bhattacharjee B, et al. Mechanism of melatonin protection against copper-ascorbate-induced oxidative damage in vitro through isothermal titration calorimetry. Life Sci 2017;180:123136. DOI: $10.1016 /$ j.Ifs.2017.05.022.

26. Reiter RJ, Mayo JC, Tan DX, et al. Melatonin as an antioxidant: under promises but over delivers. J Pineal Res 2016;61(3):253-278. DOI: 10.1111/jpi.12360.

27. Ressmeyer A-R, Mayo JC, Zelosko V, et al. Antioxidant properties of the melatonin metabolite $\mathrm{N} 1$-acetyl-5-methoxykynuramine (AMK): scavenging of free radicals and prevention of protein destruction. Redox Rep 2003;8(4):205-213. DOI: 10.1179/ 135100003225002709.

28. Li J-H, Yu J-P, Yu H-G, et al. Melatonin reduces inflammatory injury through inhibiting NF-KB activation in rats with colitis. Mediators Inflamm 2005;2005(4):185-193. DOI: 10.1155/MI.2005.185.

29. Murakami Y, Yuhara K, Takada N, et al. Effect of melatonin on cyclooxygenase-2 expression and nuclear factor-kappa B activation in RAW264.7 macrophage-like cells stimulated with fimbriae of Porphyromonas gingivalis. In Vivo 2011;25:641-647.Im Internet: http:// www.ncbi.nlm.nih.gov/pubmed/21709008.

30. Reiter RJ. Cytoprotective properties of melatonin: presumed association with oxidative damage and aging. Nutrition 1998;14(9):691-696. DOI: 10.1016/S0899-9007(98)00064-1.

31. Kang H, Lee M-J, Park S, et al. Lipopolysaccharide-preconditioned periodontal ligament stem cells induce M1 polarization of macrophages through extracellular vesicles. Int J Mol Sci 2018;19(12):3843. DOI: 10.3390/ijms19123843. 